\title{
Long-chain Omega-3 Polyunsaturated Fatty Acids: Prospects for Introduction into Horticultural Food Plants
}

\author{
David Kyle, Scott Bingham, and Richard Radmer \\ Martek Corporation, Columbia, Md.
}

\section{Introduction}

Recent data indicate that significant health benefits may be attributable to the dietary uptake of long-chain omega-3 polyunsaturated fatty acids, particularly eicosapentaenoic acid (EPA) and docosahexaenoic acid (DHA). In this paper we describe current data relating lo the nutritional significance of these compounds, their oc currence in nature and in the human food chain, data related to their biosynthesis, and the prospects for their introduction into horticultural food plants.

EPA and DHA are members of a family of fatty acids, designat ed collectively as "omega-3" (also notated as n-3) fatty acids, sodesignated because the first of the recurrent double bonds is three carbons from the methyl terminal. This general class of compounds is often referred to as PUFAs ("polyunsaturated fatty acids"), while the 20 and 22 carbon members of this group are sometimes referred to as HUFAs ("highly unsaturated fatty acids").

$$
\begin{gathered}
\mathrm{CH}_{3}-\left(\mathrm{CH}_{2}+\mathrm{CH}=\mathrm{CH}-\right)_{5}-\left(\mathrm{CH}_{2}\right)_{3}-\mathrm{COOH} \\
\Delta 5,8,11,14,17 \text { Eicosapentaenoic acid (EPA) } \\
\left.\mathrm{CH}_{3}-\left(\mathrm{CH}_{2}-\mathrm{CH}=\mathrm{CH}-\right)_{6}-\mathrm{CH}_{2}\right)_{2}-\mathrm{COOH} \\
\Delta 4,7,10,13,16,19 \text { Docosahexaenoic acid (DHA) }
\end{gathered}
$$

\section{Nutritional significance}

EPA. During the past decade. evidence has been obtained for a strong correlation between the dietary uptake of EPA and a variety of health-related effects. For example, ingestion of EPA correlates with a reduced incidence of heart disease and the relief of symptoms of rheumatoid arthritis. This compound also has shown promise for the treatment of hypercholesteremia. hypertriglyc eridemia, diabetes, and several different carcinomas.

Table 1 is a compilation of some reports relating the dietary uptake of EPA to various physiological states and diseases. Although this compilation is not exhaustive, and some of the results are controversial, this compilation does give some indication of the degree of interest by the scientific community and the potential beneficial effects of this compound. For example, epidemiological studies of the incidence of coronary (heart) disease in Greenland Eskimos and coastal Japanese have suggested that the rarity of the disease in those populations is correlated with the consumption of substantial quantities of fish, whale, and seal, which are rich in omega-3 HUFAs (Kromhout et al.. 1985; Bang and Dyerberg, 1972). The diets of Americans, on the other hand, are rich in cholesterol and the saturated fatty acids associated with animal fats or the omega- 6 PUFAs of vegetable oils. Several clinical investigations have compared the effects of diets rich in marine oils to those rich in vegetable oils under controlled conditions (Harris et al., 1983; Phillipson et al., 1985) and, in most cases, normallipidemic subjects with a fish oil-supplemented diet exhibited as significant decrease $(30 \%$ to $60 \%)$ in plasma cholesterol and triglycerides. Hyperlipidemic patients exhibited even greater reductions in plasma cholesterol and triglyceride levels (50\% to 80\%). Furthermore, the plasma lipoprotein cholesterol, which declined with the fish oil-enriched diet, was VLDL- and LDL cholesterol (the "bad cholesterol").

On a more fundamental level. there is an increasing understanding of the interrelationship between dietary fats and eicosanoids. powerful physiological mediators that include prostaglandins, thromboxanes, leucotrienes, and other related derivatives. Recent studies have shown that there are close structural and metabolic relationships between several dietary fatty acids, including EPA, and the eicosanoids (reviewed in Lands, 1987). Again, although the last word has yet to be written on this topic, there are good reasons to conclude that the dietary uptake of specific fatty acids-and. in par ticular. HUFAs - can have a significant effect on a wide variety of metabolic pathways and physiological conditions.

DHA. Docosahexaenoic acid is the most abundant PUFA in grey matter tissue in the brain (O'Brien and Sampson, 1965), and also is found in high abundance in the outer rod segments of the retina (Anderson, 1970). Evidence to dare indicates that DHA has a very significant role in human physiology and development; research is currently underway to assess the efficacy of DHA supple ments in infants. Although the role of DHA is not well-understood, this preferential association with neuronal tissues, and the modifica tion of the membrane properties imparted by this fatty acid, indicate a role in membrane excitation (Chacko et al., 1977).

DHA is tenaciously retained by neuronal tissues in which it occurs, and abnormal or pathological states associated with its depletion have been documented (see review by Salem et al., 1986). For example, epidemiological data have been compiled and reported that are consistent with the idea that multiple sclerosis is related to a dietary deficiency of HUFAs. particularly DHA or its precursors, during a critical period of neurological development. In another area, clinical studies have indicated that a congenital defect known as "Kinky Hair Disease". which results in severe mental retardation and neurological impairment in infants, correlates with a marked depression of DHA in the brain lipids. Although most of the data to date relating DHA to human health are incomplete (and in many cases indirect). there seems to be a growing body of evidence link ing the dietary uptake of significant amounts of DHA (or its metabolic precursors) with normal neurological development.

Mammals have the ability to elongate and desaturate linolenic acid to DHA. However, several lines of evidence has suggested that this conversion may not occur very rapidly in humans, and that

Table 1. Some reported effects of dietary EPA uptake.

\begin{tabular}{ll}
\hline \hline Disease or condition & Effect \\
\hline Ischaemic heart disease & Lower incidence \\
Cerebrovascular disease & Lower incidence \\
Atherosclerosis (animal model) & $80 \%$ reduction \\
Blood pressure & Small hypotensive effect? \\
Plasma triglycerides & Lowers concentrations \\
Blood cholesterol (also HDL, LDL) & Small mixed effects? \\
Arthritis (animal model) & Lessens severity \\
Cyclosporin-induced nephrotoxicity & Protection \\
Autoimmune disease & Reduced severity \\
Turnor growth & Decrease in incidence, \\
& growth, and metastatis \\
Colon cancer & Lower incidence \\
Pacreatic neoplasms & Inhibits \\
Breast cancer & No effect?
\end{tabular}

Summarized from Lands (1987). 
DHA found in human organs probably originates from DHA in the diet. This idea is supported by [he observation that relatively small amounts of 22:5 or 22:6 HUFA dietary supplements alter tissue composition more significantly than large amounts of linolenic acid (18.3). the biosynthetic precursor (Sanders and Younger, 1981; Dyerberg et al.. 1980). Further supporting this concept are the reports that infants whose diet is DHA-deficient, either from formula feeding or from mothers adhering to a strict vegetarian diet. exhibit DHA levels in specific lipid fractions that are reduced by as much as 50\% compared to normal breast-fed babies (Putnam et al., 1982). and that infants fed only cow's milk (which is deficient in DHA compared to human milk) can show significant symptoms of an es sential fatty acid deficiency. This apparent importance of DHA in the human diet led Crawford (1987) to hypothesize that "the availability of docosahexenoic acid within the food chain and made available during fetal development could have been a controlling factor in the evolution of the brain and its sophisticated attributes".

\section{Occurrence in nature}

The most generally recognized source of HUFAs is the flesh and oil of certain marine finfish. However. although it is not generally realized, the phytoplankton in [he ocean represent by far the largest depot of EPA and DHA on [his planet (see for example. Laskin and Lechevalier, 1978). Like humans, the fish from which the omega-3 HUFA-rich oil is extracted are probably bioaccumulators, while it is the phytoplankton that are the ultimate producers.

Table 2 is a compilation of some data obtained in our laboratory in which we assessed the ability of minoalgae to produce and accu mulate fatty acids, particularly EPA and DHA. These organisms include both diatoms and dinoflagellates obtained directly from culture collections. and there was no effort to optimize lipid or HUFA contents. Many oleagenous microalgae have been reported to make oil in response to a nutrient deficiency such as nitrogen, phosphate, or. in the case of diatoms, silica (Wheeler, 1982; Kyle et al.. 1988). Thus, we might expect that organisms of this type would, under some natural conditions, be prolific producers of these HUFAs in nature. Substantial quantities of EPA (but not DHA) also have been found in some species of fungi (Gellerman and Schlenk, 1978). and these organisms also may prove to be valuable sources of genetic determinants governing HUFA biosynthesis.

In contrast to [he cases discussed above, EPA and DHA are rarely, if ever. found in most of the rest of the plant and animal kingdoms. An extensive compilation of data assembled by the U.S. Department of Agriculture (see Simopolous et al.. 1986) showed that, with the exception of fish and other seafood, no appreciable EPA or DHA is present in beef, lamb, pork. poultry, dairy and egg products. cereals, legumes, or fruits and vegetables.

\section{HUFA biosynthesis: Precursors and pathways}

To date, there are little available data concerning the pathways for the biosynthesis of EPA and DHA in microalgae and fungi. Algae of the Chlorophyceae (the so-called "green algae") generally produce oils with fatty acid compositions similar to chose of higher plants and yeasts (i.e.. predominantly palmitic, oleic, linoleic. and linolenic acids) and the pathways of fatty acid biosynthesis in these organisms appear to be remarkably similar to those of higher plants (Erwin, 1973). In both taxonomic groups, the biosynthesis of palmitic and stearic acids occurs on a multi-enzyme fatty acid synthetase complex via serial condensation reactions in a compartmentalized system similar to that used by higher plants (Stumpf, 1984).

Several pathways of fatty acid desaturation have been described in microalgae. Many of the Chlorophyceae (e.g., Chlorella) use a soluble slearate desaturase system, similar to that of higher plants, and produce an oil rich in oleic acid (Nagal and Bloch, 1968). The Bacillariophyceae (Diatoms), on the other hand, appear [o have an active palmitate desaturase system, [hereby generating a large amount of palmitoleic acid in their oil (Behrens et al., 1989). In both cases the initial desaturation is at the $\Delta 9-10$ position, with the further elongation and desaturation reactions varying from species to species. The elongases may be soluble enzymes; the desaturases appear to involve a more complex, membrane-associated series of reactions that may use various substrate forms of the fatty acids (Stumpf, 1984).

As alluded to above, [he actual metabolic pathways and reaction mechanisms leading to EPA and DHA are poorly understood. A general survey indicates that there are at least three pathways of polyunsaturated fatty acid biosynthesis in microalgae. although none of these to date have been demonstrated to specifically gener ate EPA or DHA. The pathways to these HUFAs can be considered as connecting elements of a "HUFA biosynthesis matrix" (see Fig. 1), with biosynthesis progressing from the upper-left to the lowerright via a series of desaturase reactions (progressing to the right) and elongate reactions (moving to the bottom). The specific pathways will then reflect obligate or preferred transitions within this matrix. beginning with la-carbon precursors generally found in higher plants. For example, Mead and Willis (1988) provided evidence for a "consensus pathway" to arachidonic acid. (20:4. omega6 ) that proceeds via the route 18:3 $\rightarrow 18.4 \rightarrow 20: 4$. Pohl(1982) suggested that the biosynthesis of arachidonic acid can take place by the two pathways leading from 18:2 to $20: 3$ (i.e., 18:2 $\rightarrow 18: 3$ $\rightarrow 20: 3$ and 18:2 $\rightarrow$ 20:2 $\rightarrow$ 20:3), with the first pathway predominating in several different algal classes (as well as in animals) and the second predominating in the Euglenophyceae. One can speculate that the multipath matrix of Fig. 1 has some biochemical basis, and that even within a particular organism HUFA synthesis

Table 2. Fatty acid composition of selected microalgae.

\begin{tabular}{lcccc}
\hline Fatty acid & Chaetoceros sp. & $\begin{array}{c}\text { Skeletonema } \\
\text { costatum }\end{array}$ & $\begin{array}{c}\text { Gonyaulx } \\
\text { diagensis }\end{array}$ & $\begin{array}{c}\text { Gymnodinium } \\
\text { splendens }\end{array}$ \\
\hline $14: 0$ & 6.7 & 7.9 & 3.5 & 4.5 \\
$16: 0$ & 15.0 & 9.2 & 8.8 & 10.3 \\
$16: 1$ & 21.1 & 39.0 & 8.8 & 9.7 \\
$18: 0$ & 1.9 & 8.6 & 10.2 & 8.7 \\
$18: 1$ & 6.9 & 8.8 & 9.9 & 9.7 \\
$18: 2$ & 7.0 & 4.5 & - & 1.8 \\
$18: 3$ & 2.9 & 4.6 & 11.9 & 13.9 \\
$18: 4$ & 5.4 & 1.0 & 9.7 & 6.0 \\
$20: 4$ & 7.8 & -- & 6.0 & -.8 \\
$20: 5$ EPA & 4.9 & 6.7 & -- & 6.0 \\
$22: 4$ &.- & -- & 7.3 & 3.4 \\
$22: 5$ & 2.2 & -- & 3.8 & 19.2 \\
$22: 6$ DHA & 15.4 & 3.7 & 13.8 & \\
\hline
\end{tabular}

Some common names: 14:0. Myristic; 16:0, Palmitic; 18:0, Stearic; 18:1, Oleic: 18:2, Linoleic: 18:3. Linolenic. 
can follow more than one pathway. This idea is supported by the observation that the $\Delta 5$ desaturase of Saprolegnia (a marine fungus), which is considered to normally convert $20: 3$ into arachidonic acid (20:4, omega-6), also converts the omega-3 20:4 into EPA (20:5. omega-3). The C- 18 elongase in this organism also is non specific. and will elongate both 18:4 and 18:3 (Gellerman and Schlenk. 1978).

\section{Prospects for the introduction of HUFAs into horticultural food plants}

The preceding discussion shows that some microalgae, which as a group can be considered to be "microplants". have the HUFAs EPA and DHA as significant fatty acid components. Furthermore, these HUFAs appear to be synthesized by straightforward extensions of existing higher plant fatty acid pathways, and appear to use fatty acid precursors that may exist in many (or possibly all) higher plants. Thus. these organisms provide a potential source of genetic determinants for specifying the production of EPA and DHA.

Table 3 shows the content of unsaturated 18-carbon fatty acids in the oil of several higher plants of potential interest. Note that in all cases these fatty acids, which are precursors of EPA and DHA according to the scheme of Fig. 1, are present in very significant quantities; in fact, they comprise the majority of the total fatty acids present in the oil of these plants. Since the evidence to date suggests that the plant and fungal enzymes can use these existing plant fatty acids precursors, the introduction of "HUFA genes" (i.e., spe cific desaturases and elongases) inro these horticultural food plants could result in EPA- or DHA- containing plant oils.

The following major steps would be required to develop a horti cultural plant that produces an oil containing EPA and DHA:

1) Identify and isolate the enzymatic activities involved in the synthesis of EPA and DHA. This activity is quite straightforward, and makes use of standard biochemical techniques. Preliminary work in our laboratory has resulted in the successful isolation and solubilization of the polyunsaturated fatty acid elongase and the identification of weak $\Delta 6-$ desaturase activity. These initial successes provide some measure of confidence that the biochemical as pects of this project could be completed in a reasonable period of time. The "final product" of this activity would be a suite of puri fied enzymes that have been characterized by chromatographic pro cedures and antibody screening.

2) Use these enzyme activities to isolate the corresponding genes from an algal genomic library. Unlike step 1 above, the pro cedures required for this activity are quite new and have only been in general use for a few years. This activity would involve a) preparation of a so-called "cDNA library" of the mRNAs of the subject organism. b) screening of this cDNA library using antibody prepared to the purified subject enzyme, c) identification of positive

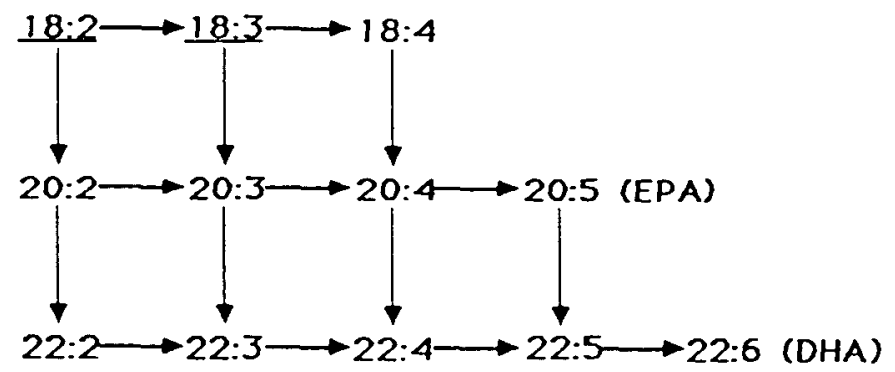

Fig. 1. HUFA biosynthesis matrix, which schematically represents the observed and potential biosynthetic pathways from linoleic acid (18:2) and linolenic acid (18:3). two presursors present in higher plants (underlined). to EPA (20:5) and DHA (22:6). Note that some of these fatty acids may exist in more than one isomeric form; e.g., omega-3 18:3 and omega-6 18:3.
Table 3. Unsaturated 18-carbon fatty acid composition of selected plant oils $(\%)$.

\begin{tabular}{llll}
\hline Plant & $18: 1$ & $18: 2$ & $18: 3$ \\
\hline Olive & 70 & 13 & 0.5 \\
Palm & 40 & 10 & 0.2 \\
Peanut & 52 & 27 & - \\
Rapeseed & 63 & 20 & 9 \\
Sunflower & 20 & 68 & 0.5 \\
\hline
\end{tabular}

Adapted from Zabriskie et al. (1980).

clones (that contain the true subject enzyme genes) using a partial amino acid sequence determined from the purified protein, and d) complete sequencing of the subject enzyme gene.

3) Develop genetic transformation and expression systems in the targeted plant. The projected time frame and the level-of-effort required for this activity could vary widely, depending on the target plant. For example, a genetic transformation system for canola (rapeseed) is currently available (Horsch et al.. 1988).

4) Transform the targeted plant with the algal genes and obtain useful gene expression (i.e., EPA or DHA synthesis). This step could prove to be the major hurdle. The expression and regulation of an introduced multistep biosynthetic pathway is currently at the leading edge of biotechnology.

\section{Summary}

Clinical and epidemiological data link the dietary uptake of EPA and DHA to significant health benefits. The dietary uptake of EPA correlates with a reduced incidence of heart disease and the relief of rheumatoid arthritis symptoms; this compound also is being tested for the treatment of hypertriglyceridemia and several carcinomas. DHA is an important component of brain and visual tissue, and may prove to be a vital nutrient during infant development.

EPA and DHA are synthesized by microalgae, which are members of the plant kingdom. However, no higher plants are currently capable of producing these compounds. EPA and DHA appear to be synthesized by extensions of pathways presently available in higher plants using precursors also available in higher plants. Thus, provided that there is sufficient economic justification, the development of horticultural oil-producing crop plants whose oil contains significant quantities of EPA and/or DHA appears to be an attainable goal.

\section{References}

Anderson, R.E. 1970. Expt. Eye Res. 10:339.

Behrens. P.W., S.D. Hoeksema, K.L Amen, M.S. Cole, T.A. Heubner, J.M. Rutten, and D.J. Kyle. 1989. Novel microbial product for medicine and agriculture. Elsevier, Amsterdam. p. 253.

Bang H. and J. Dyer-berg. 1972. Acta Med. Scan 192:85

Chacko,. G.K., F.V. Barnola, and R. Villegas. 1977. J. Neurochem. 28:445.

Crawford. M.A. (1987) "Proc. AOCS Short Course on Polyunsaturated Fatty Acids and Eicosanoids. Amer. Oil Chemists' Soc., Champaign, Ill., p. 270.

Dyerberg, J.. H. Bang, and O. Aagaard. 1980. Lancet i:199.

Erwin. J. 1973. Lipids and biomembranes of eucaryotic microorganisms". Academic, New York. p. 41.

Gellerman, J.L and H. Schlenk. 1978. Biochim. Biophys. Acta 573:23.

Harris, W.S.. W.E. Connor, and M.P. McMurry. 1983. Metabolism 32:179.

Horsch, R.B., J.E. Fry, A. Bamason. S. Merz. S.G. Rogers, and R.T. Fraley. 1988. Proc. World Con. on Biotechnology for the Fats and Oils Industry" American Oil Chemists' Soc. p. 43.

Kromhous, D.. E.B. Bosschieter. and C. Coulander. 1985. New Engl. J. Med. 321:1205.

Kyle. D.J., P.W. Behrens. S. Bingham. K. Amen. and D. Lieberman 1988 Proc. World Conf. on Biotechnology for the Fats and Oils Industry". American Oil Chemists' Soc.p. 117. 
Lands, W.E.M. 1987. Proc. AOCS Short Course on Polyunsaturated Fatty Acids and Eicosanoids. American Oil Chemists' Soc., Champaign, Ill.

Laskin, A.L and H.A. Lechevaller. 1978. Handbook of Microbiology. 2nd ed. vol. II. CRC Press, West Palm Beach, Fla.

Mead, J.F. and A.L. Willis. 1988. CRC handbook of eicosanoids: Prostaglandins and related lipids. CRC Press, Boca Raton. Fla. p. 85

Nagal, J. and Bloch 1968. J. Biol. Chem. 243:4626.

O’Brien. J.S. and E.L. Sampson. 1965. J. Lipid Res. 6:545

Phillipson. B.E.. D.W. Rothrock, W.E. Connor. W.S. Harris, and D.R. Illingwirth. 1985. New Engl. J. Med. 312:1210.

Pohl, P. 1982. CRC handbook of biosolar resources. vol. 1 part 1, CRC Press, Boca Raton, Fla. p. 383.

Putnam, J.C.. S.E. Carlson. P.W. DeVoe, and LA. Barness. 1982. Amer. J. Clin. Nutr. 35: 106.
Salem. N.. H.-Y. Kim, and J.A. Yergey. 1986. Health Effects of Polyunsaturated Fatty Acids in Seafoods. Academic. Orlando, Fla. p. 263.

Sanders, T.A.B. and K.M Younger. 1981. Brit. J. Nutr. 45:613.

Simopoulos, A.P., R.R. Kifer, and R.E. Martin. 1986. Health effects of polyunsaturated fatty acids in seafoods. Academic, New York. p. 453.

Stumpf, P.K. 1984. Fatty acid metabolism and its regulations. Elsevier. New York. p. 155.

Wheeler, W.N. 1982. Handbook of biosolar resources. CRC Press, Boca Raton, Fla. p. 157.

Zabriskie. D.W.. W.B. Armiger, D.H. Phillips, and P.A. Albano, 1980. Traders' guide to fermentation media formulation. Traders Protein. Memphis, Tenn. 